\title{
Empagliflozin, an Inhibitor of Sodium-Glucose Cotransporter 2 Exerts Anti-Inflammatory and Antifibrotic Effects on Experimental Diabetic Nephropathy Partly by Suppressing AGEs-Receptor Axis
}

Authors

Affiliation

\section{A. Ojima, T. Matsui, Y. Nishino, N. Nakamura, S. Yamagishi}

Department of Pathophysiology and Therapeutics of Diabetic Vascular Complications, Kurume University School of Medicine, Kurume, Japan

\author{
Key words \\ - AGES \\ - RAGE \\ - diabetic nephropathy \\ - oxidative stress \\ - SGLT2
}

received 20.09.2014

accepted 03.11.2014

\section{Bibliography}

Dol http://dx.doi.org/

10.1055/s-0034-1395609

Published online:

January 22, 2015

Horm Metab Res 2015;

47: 686-692

(c) Georg Thieme Verlag KG

Stuttgart · New York

ISSN 0018-5043

\section{Correspondence}

\section{Dr. S.-i. Yamagishi}

Department of Pathophysiology and Therapeutics of Diabetic

Vascular Complications

Kurume University School of

Medicine

67 Asahi-machi

Kurume 830-0011

Japan

Tel.: + 81/942/31 7873

Fax: $+81 / 942 / 317895$

shoichi@med.kurume-u.ac.jp

\section{Abstract}

$\nabla$

Advanced glycation end products (AGEs) and receptor RAGE play a role in diabetic nephropathy. We have previously shown that increased glucose uptake into proximal tubular cells via sodium-glucose cotransporter 2 (SGLT2) stimulates oxidative stress generation and RAGE expression, thereby exacerbating the AGEinduced apoptosis in this cell type. However, the protective role of SGLT2 inhibition against the AGE-RAGE-induced renal damage in diabetic animals remains unclear. In this study, we investigated the effects of empagliflozin, SGLT2 inhibitor on AGE-RAGE axis, inflammatory and fibrotic reactions, and tubular injury in the kidney of streptozotocin-induced diabetic rats.

Administration of empagliflozin for 4 weeks significantly improved hyperglycemia and HbA1c, and decreased expression levels of AGEs, RAGE, 8-hydroxydeoxyguanosine (8-OHdG), and F4/80,

\section{Introduction}

$\nabla$

Diabetic nephropathy is a leading cause of endstage renal disease, which could account for disability and high mortality rate in patients with both type 1 and type 2 diabetes [1,2]. Diabetic nephropathy is characterized by functional and structural changes in the glomerulus such as glomerular hyperfiltration, thickening of glomerular basement membrane, and an expansion of extracellular matrix in the mesangial areas $[1,2]$. It ultimately progresses glomerular sclerosis associated with an increased urinary excretion rate of albumin and renal dysfunction [1,2]. In addition, although Alsaad et al. reported that the characteristic histological changes of diabetic nephropathy were diffuse and nodular glomerulosclerosis, but not renal tubular injury [3], it has been recognized that changes within tubulointerstitium are markers of oxidative stress and macrophages, respectively, in the diabetic kidney. Although empagliflozin did not reduce albuminuria, it significantly decreased urinary excretion levels of 8-OHdG and L-fatty acid binding protein, a marker of tubular injury. Moreover, inflammatory and fibrotic gene expression such as monocyte chemoattractant protein-1, intercellular adhesion molecule-1, plasminogen activator inhibitor-1, transforming growth factor- $\beta$, and connective tissue growth factor was enhanced in the diabetic kidney, all of which were prevented by empagliflozin. The present study suggests that empagliflozin could inhibit oxidative, inflammatory and fibrotic reactions in the kidney of diabetic rats partly via suppression of the AGE-RAGE axis. Blockade of the increased glucose uptake into renal proximal tubular cells by empagliflozin might be a novel therapeutic target for tubulointerstitial damage in diabetic nephropathy.

more important than glomerulopathy in terms of renal dysfunction in diabetic nephropathy $[4,5]$. Nonenzymatic modification of proteins by reducing sugars, a process that is also known as Maillard reaction, progress at an extremely accelerated rate under diabetic conditions [6-8]. These early glycation products undergo further complex reaction, such as rearrangement, dehydration, and condensation, to become irreversibly cross-linked, heterogeneous fluorescent derivatives, termed advanced glycation end products (AGEs) [6-8]. There is an accumulating body of evidence that engagement of receptor for AGEs (RAGE) with the ligand AGEs elicits oxidative stress generation and resultantly evokes inflammatory and fibrotic reactions in the kidney cells, thereby causing progressive alteration in renal architecture and loss of renal function associated with tubular injury in diabetes [9-16]. 
Therefore, the AGE-RAGE-induced renal damage might be a therapeutic target for diabetic nephropathy.

Ninety percent of glucose filtered by the glomerulus is reabsorbed by a low-affinity/high capacity sodium-glucose cotransporter 2 (SGLT2), which is expressed mainly on the apical membrane of renal proximal tubules [17-19]. SGLT2 is expressed in the $\mathrm{S} 1$ and $\mathrm{S} 2$ segment of the proximal tubule but not S3 segment $[18,19]$. Since blockade of SGLT2 promotes urinary glucose excretion and resultantly improves hyperglycemia in an insulinindependent manner, selective inhibition of SGLT2 has been proposed as a potential therapeutic target for the treatment of patients with diabetes [20]. We have previously shown that increased glucose uptake into cultured renal proximal tubular cells via SGLT2 stimulates oxidative stress generation and RAGE expression, thereby potentiating the pro-apoptotic effects of AGEs on tubular cells [21]. Furthermore, we found in the previous study that an antioxidant, $\mathrm{N}$-acetylcysteine or small interfering RNAs raised against SGLT2 (siSGLT2) blocked the harmful effects of high glucose on AGE-exposed renal proximal tubular cells [21]. These observations suggest that suppression of SGLT2 might have beneficial effects on diabetic nephropathy. However, the protective role of SGLT2 inhibition against the AGE-RAGEinduced renal damage in experimental diabetic nephropathy remains unclear. Therefore, in this study, we investigated the effects of empagliflozin, a selective inhibitor of SGLT2 on AGEs and RAGE expression, oxidative stress generation, inflammatory and fibrotic reactions, and tubular injury in the kidney of streptozotocin-induced diabetic rats (STZ rats).

\section{Materials and Methods}

$\nabla$

Materials

A selective inhibitor of SGLT2, empagliflozin was a generous gift from Boehringer Ingelheim (Ingelheim, Germany). Bovine serum albumin (BSA) (essentially fatty acid-free and essentially globulin-free, lyophilized powder) was purchased from Sigma (St. Louis, MO, USA). D-Glyceraldehyde was obtained from Nakalai Tesque (Kyoto, Japan).

\section{Animal experiments}

Six-week-old male Spraque-Dawley (SD) rats received single $60 \mathrm{mg} / \mathrm{kg}$ intraperitoneal injection of streptozotocin (Sigma, St. Louis, MO, USA) in $10 \mathrm{mM}$ citrate buffer (pH 4.5). Nondiabetic control rats (Control) received citrate buffer alone. Animals with blood glucose levels greater than $250 \mathrm{mg} / \mathrm{dl} 48 \mathrm{~h}$ later were considered diabetic. STZ rats received $10 \mathrm{mg} / \mathrm{kg}$ empagliflozin or vehicle by oral gavage. At baseline and/or after 4 weeks of treatment, animals were housed in metabolic cages to collect urine for measurement of urinary excretion levels of glucose, albumin, 8-hydroxydeoxyguanosine (8-OHdG), and L-fatty acid binding protein (L-FABP), and then body weight, heart rate, blood pressure (BP), fasting blood glucose, and blood biochemistries were measured. BP was monitored by a tail-cuff sphygmomanometer (BP-98A; Softron, Tokyo, Japan). Urinary excretion levels of 8-OHdG (Japan Institute for the Control of Aging NIKKEN SEIL Co., Ltd., Shizuoka, Japan), albumin (AssayPro, Charles, MO, USA) and L-FABP (R\&D systems, Minneapolis, MN, USA) were determined with commercially available enzyme-linked immunosorbent assay (ELISA) kits. Other biochemistries were determined as described previously [14]. Then, the rats were killed and the kidneys were excised for real-time reverse transcription-poly- merase chain reactions (RT-PCR), western blotting and immunohistochemical analyses. All experimental procedures were conducted in accordance with the National Institutes Health Guide for Care and Use of Laboratory Animals and were approved by the ethical committee of Kurume University School of Medicine.

\section{Western blotting analysis}

Five micrograms of proteins were extracted from the kidneys of Control, STZ or STZ rats + empagliflozin with lysis buffer, and then separated by SDS-PAGE and transferred to nitrocellulose membranes as described previously [14]. Membranes were probed with antibodies (Abs) raised against AGEs or $\alpha$-tubulin (Santa Cruz Biotechnology Inc., Delaware, CA, USA), and then immune complexes were visualized with an enhanced chemiluminescence detection system (Amersham Bioscience, Buckinghamshire, UK) as described previously [14].

\section{Preparation of AGE-BSA and Abs raised against AGEs}

AGE-BSA was prepared as described previously [22]. In brief, BSA $(25 \mathrm{mg} / \mathrm{ml})$ was incubated under sterile conditions with $0.1 \mathrm{M}$ glyceraldehyde in $0.2 \mathrm{M} \mathrm{NaPO}_{4}$ buffer ( $\mathrm{pH} 7.4$ ) for 7 days. Then, unincorporated sugars were removed by PD-10 column chromatography and dialysis against phosphate-buffered saline. Control nonglycated BSA was incubated in the same conditions except for the absence of reducing sugars. Preparations were tested for endotoxin using Endospecy ES-20S system (Seikagaku Co., Tokyo, Japan); no endotoxin was detectable. Abs raised against AGEs were prepared as described previously [22].

\section{RT-PCR}

Total RNAs were extracted from the kidneys of Control, STZ or STZ rats + empagliflozin with RNAqueous-4PCR kit (Ambion Inc., Austin, TX, USA) according to the manufacturer's instructions. Quantitative real-time RT-PCR was performed using Assay-onDemand and TaqMan 5 fluorogenic nuclease chemistry (Applied Biosystems, Foster city, CA, USA) according to the supplier's recommendation. IDs of primers for rat RAGE, monocyte chemoattractant protein-1 (MCP-1), intercellular adhesion molecule-1 (ICAM-1), plasminogen activator inhibitor-1 (PAI-1), transforming growth factor- $\beta$ (TGF- $\beta$ ), connective tissue growth factor (CTGF), SGLT1, SGLT2, and 18S gene were Rn00584249_ m1, Rn00580555_m1, Rn00564227_m1, Rn01481341_m1, Rn99 999016_m1, Rn00573960_g1, Rn01640634_m1, Rn00574917_ m1, and Hs99999901_s1, respectively.

\section{Immunohistochemical analysis}

The kidneys were fixed in $4 \%$ paraformaldehyde and embedded in paraffin, sectioned at $4 \mu \mathrm{m}$ intervals and mounted on glass slides. After blocking endogenous peroxidase activity, the sections were incubated overnight at $4{ }^{\circ} \mathrm{C}$ with anti-RAGE Abs (Santa Cruz Biotechnology Inc.), anti-8-OHdG Abs (Japan Institute for the Control of Aging NIKKEN SEIL Co., Ltd.) or anti-F4/80 Abs directed specially against macrophages (Santa Cruz Biotechnology Inc.). Then, the reactions were visualized with a Histofine Simple Stain Rat MAX-POMULTI kit (Nichirei Co., Tokyo, Japan) as described previously [14]. Immunoreactivity of each sample was measured by microcomputer-assisted image J.

\section{Statistical analysis}

All values were presented as means \pm standard error. One-way analysis of variance followed Tukey's test was performed for statistical comparisons; $\mathrm{p}<0.05$ was considered significant. 


\section{Results}

$\nabla$

As shown in 0 Table 1, compared with Control rats at 10 week old, heart rate, and serum creatinine levels were significantly lower, while glycated hemoglobin (HbA1c), albuminuria and glucosuria were higher in diabetic rats (STZ rats). Moreover, body weight was significantly lower, whereas fasting blood glucose values were significantly higher in STZ rats at 8 and 10 week old compared with Control rats at the same age, respectively ( $\odot$ Fig. 1). Although a selective inhibitor of SGLT2, empagliflozin treatment did not affect body weight, heart rate, serum creatinine, albuminuria, or glucosuria in STZ rats, it significantly decreased HbA1c and fasting blood glucose values in these animals ( $\bullet$ Table 1 and $\odot$ Fig. 1 ).

We next investigated the effects of empagliflozin on AGE-RAGEoxidative stress axis in the kidney of diabetic rats at 10-week old. As shown in $\odot$ Fig. 2 and $\odot$ Fig. 3a, AGE-modified protein levels, RAGE gene and protein expression and a marker of oxidative stress, 8-OHdG levels were significantly increased in the kidney of type 1 diabetic rats compared with nondiabetic control rats, all of which were completely blocked by the treatment with empagliflozin. Moreover, empagliflozin treatment for 4 weeks significantly inhibited macrophage infiltration into the glomeruli and decreased urinary excretion levels of $8-O H d G$ and L-FABP, a marker of tubular injury in STZ rats at 10 -week old ( $\odot$ Fig. $\mathbf{3 b}-\mathbf{d}$ ). We further examined the effects of empagliflozin on inflammatory and fibrotic gene expression in the kidney of diabetic rats. As shown in 0 Fig. 4a-e, empagliflozin treatment for 4 weeks significantly inhibited the increase in MCP-1, ICAM-1, PAI-1, TGF- $\beta$, and CTGF mRNA levels in the kidney of diabetic rats. In addition, although there were no significant differences of SGLT2 gene expression among Control, STZ and STZ rats + empagliflozin groups, renal SGLT1 mRNA levels were increased in STZ rats, which were also prevented by the treatment with empagliflozin (๑ Fig. 4f, g).

\section{Discussion}

$\nabla$

We have recently found that, (1) high glucose induces oxidative stress generation and RAGE protein expression in human cultured proximal tubular cells, (2) high glucose augments AGE- induced apoptotic cell death of tubular cells, (3) siSGLT2 transfection reduces SGLT2 expression in tubular cells and subsequently blocks the entry of glucose into the cells, (4) suppression of SGLT2 expression by siSGLT2 or an antioxidant, $\mathrm{N}$-acetylcysteine inhibits high glucose-elicited oxidative stress generation and RAGE induction, and (5) pro-apoptotic effects of high glucose in AGE-exposed tubular cells are blocked by siSGLT2 treatment [21]. These observations suggest that SGLT2-

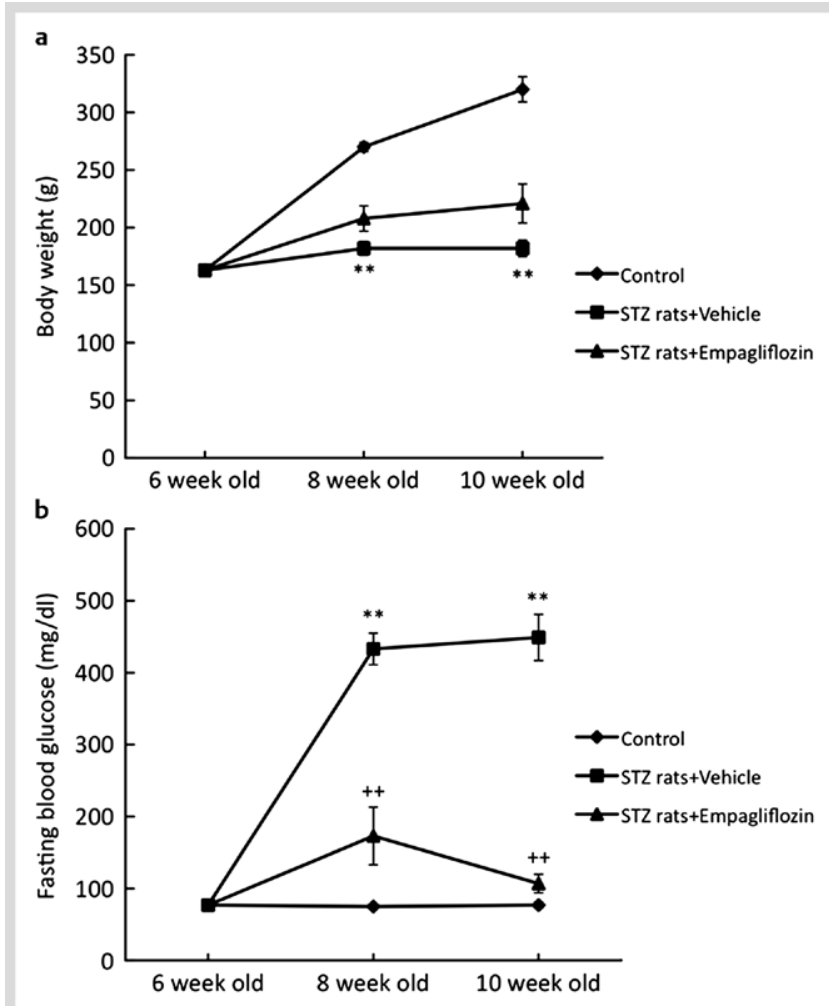

Fig. 1 Effects of empagliflozin on body weight a and fasting blood glucose $\mathbf{b}$ in Control, STZ and STZ rats + empagliflozin. Diamond: Control rats; Square: STZ rats with vehicle treatment; Triangle: STZ rats with empagliflozin treatment. ${ }^{*}{ }^{*} p<0.01$ compared to the value with Control rats. $++p<0.01$ compared to the value with STZ rats with vehicle treatment. $n=5$ for Control rats. $n=6$ for STZ rats + vehicle. $n=7$ for STZ rats + empagliflozin.

Table 1 Characteristics of animals.

\begin{tabular}{|c|c|c|c|c|}
\hline Parameters & $\begin{array}{l}\text { 6-Week old } \\
\text { SD rats }\end{array}$ & $\begin{array}{l}\text { 10-Week old } \\
\text { Control }\end{array}$ & STZ rats + Vehicle & STZ rats + Empagliflozin \\
\hline Number & 4 & 5 & 6 & 7 \\
\hline Heart rate (beats per min) & $429.4 \pm 11.6$ & $367.8 \pm 10.4$ & $287.2 \pm 4.9^{* *}$ & $333.2 \pm 31.2$ \\
\hline Systolic blood pressure $(\mathrm{mm} \mathrm{Hg})$ & $126.2 \pm 2.3$ & $120.6 \pm 5.6$ & $126.1 \pm 4.3$ & $147.0 \pm 6.4$ \\
\hline Diastolic blood pressure $(\mathrm{mm} \mathrm{Hg})$ & $77.3 \pm 8.5$ & $87.0 \pm 4.8$ & $70.1 \pm 8.6$ & $92.7 \pm 9.3$ \\
\hline HbA1c (mmol/mol) & - & $31 \pm 3$ & $79 \pm 7^{* *}$ & $62 \pm 6^{++}$ \\
\hline $\mathrm{HDL}-\mathrm{C}(\mathrm{mg} / \mathrm{dl})$ & $66.0 \pm 5.2$ & $46.8 \pm 2.7$ & $46.0 \pm 4.0$ & $36.4 \pm 5.1$ \\
\hline Total cholesterol (mg/dl) & $82.9 \pm 6.0$ & $64.5 \pm 2.9$ & $58.3 \pm 7.1$ & $48.9 \pm 4.6$ \\
\hline Triglycerides (mg/dl) & $104.6 \pm 4.8$ & $57.7 \pm 9.1$ & $109.6 \pm 62.9$ & $30.4 \pm 8.1$ \\
\hline Creatinine $(\mathrm{mg} / \mathrm{dl})$ & $0.14 \pm 0.02$ & $0.22 \pm 0.02$ & $0.15 \pm 0.02^{*}$ & $0.15 \pm 0.02$ \\
\hline Albuminuria (mg/day) & $0.05 \pm 0.01$ & $0.09 \pm 0.02$ & $0.18 \pm 0.01^{* *}$ & $0.29 \pm 0.09$ \\
\hline Glucosuria (mg/day) & $1.5 \pm 0.4$ & $1.6 \pm 0.4$ & $13149.2 \pm 487.7^{* *}$ & $13834.6 \pm 1783.7$ \\
\hline
\end{tabular}

Data are shown as mean \pm standard error

STZ: strepotozotocin-induced diabetic rats; HbA1c: Glycated hemoglobin; HDL-C: High-density lipoprotein cholesterol

* and ${ }^{*}{ }^{*} p<0.05$ and $p<0.01$ compared with the values of Control at the same age, respectively

${ }^{++} \mathrm{p}<0.01$ compared with the values of STZ rats + Vehicle at the same age 


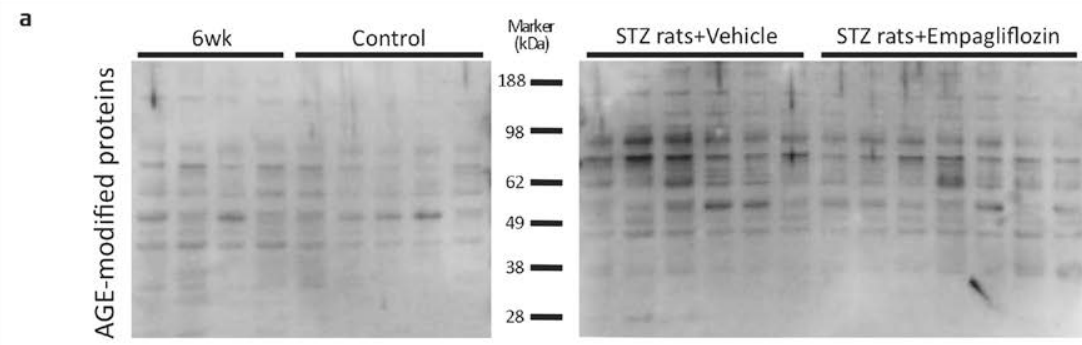

$\alpha$-tubulin -------
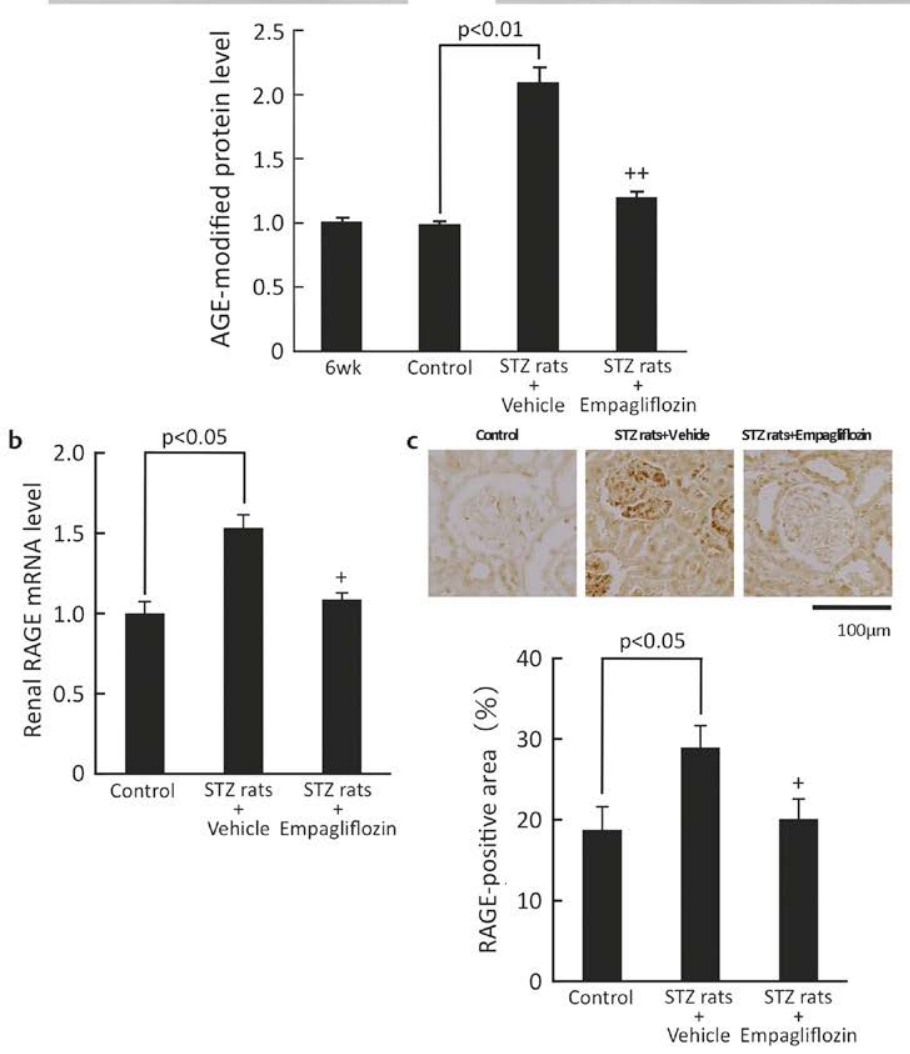

Fig. 2 Effects of empagliflozin on AGE-modified protein levels $\mathbf{a}$, RAGE gene expression $\mathbf{b}$, and RAGE immunostaining $\mathbf{c}$ in the kidney of Control, STZ and STZ rats + empagliflozin. a Five micrograms of proteins were extracted from the kidney of Control, STZ and STZ rats + empagliflozin with lysis buffer, and then separated by SDS-PAGE and transferred to nitrocellulose membranes. Membranes were probed with Abs raised against AGEs, and then immune complexes were visualized with an enhanced chemiluminescence detection system. Upper panel shows the representative bands of Western blots. Lower panel shows the quantitative data. Data were normalized by the intensity of $\alpha$-tubulin-derived signals and related to the value of 6-week-old SD rats. b Total RNAs were extracted from the kidney of Control, STZ and STZ rats + empagliflozin with RNAqueous4PCR kit. Quantitative real-time RT-PCR was performed. Data were normalized by the intensity of 185 mRNA-derived signals. c The kidneys were fixed in $4 \%$ paraformaldehyde and embedded in paraffin, sectioned at $4 \mu \mathrm{m}$ intervals and mounted on glass slides. Then the sections were incubated overnight at $4^{\circ} \mathrm{C}$ with anti-RAGE Abs, and the reactions were visualized with a Histofine Simple Stain Rat MAX-POMULTI kit. Immunoreactivity in 5 different field of each sample was measured by microcomputer-assisted image J. Upper panel shows the representative microphotograph. Lower panel shows the quantitative data. $\mathbf{a}-\mathbf{c}+$ and ++ , $p<0.05$ and $p<0.01$ compared to the value with STZ rats with vehicle treatment, respectively. $n=5$ for Control rats. $n=6$ for STZ rats + vehicle. $n=7$ for STZ rats + empagliflozin. (Color figure available online only). mediated glucose entry into the tubular cells might potentiate the cells' susceptibility toward harmful effects of AGEs via RAGE overexpression, thereby being involved in diabetic nephropathy. However, it remains unclear whether an oral selective inhibitor of SGLT2, empagliflozin has beneficial effects on renal injury in diabetic rats partly by suppressing the AGE-RAGE-evoked oxidative stress production.

In the present study, we found for the first time that, (1) empagliflozin treatment significantly decreased fasting blood glucose and HbA1c values in STZ rats at 8 and 10 week old, (2) AGEs, RAGE gene and protein expression, an oxidative stress marker, 8-OHdG levels and macrophage infiltration in the kidneys of diabetic rats were significantly increased, all of which were completely prevented by empagliflozin, (3) empagliflozin treatment for 4 weeks also completely prevented the increase in urinary excretion levels of 8-OHdG and L-FABP, a marker of tubular injury, and (4) renal inflammatory and fibrotic gene expression such as MCP-1, ICAM-1 PAI-1, TGF- $\beta$, and CTGF was increased in diabetic rats, which was blocked by empagliflozin. In this study, although effects of empagliflozin on blood glucose and HbA1c values were partial, it completely blocked the AGE-RAGE oxidative stress axis in the kidney of diabetic rats. Since proximal tubular cells are the major constituent of the kidney cortex, the present findings suggest that increased proximal tubular reabsorption of glucose via SGLT2 could directly stimulate AGEs formation, RAGE expression and oxidative stress production in the proximal tubule of diabetic kidney. Empagliflozin has been shown to reduce high glucose-induced interleukin- 6 and type 4 collagen expression in HK2 cells, a human kidney proximal tubule cell line by suppressing redox-sensitive transcriptional factor, nuclear factor-KB and activator protein-1 via blockade of glucose entry into the cells [23]. Nonspecific SGLT2 inhibitor, phlorizin prevents oxidative stress generation in the kidney of STZ rats [24]. Moreover, glucose uptake from the tubular lumen has been shown to contribute to overexpression of extracellular matrix synthesis by proximal tubular cells [25]. These observations further support the concept that glucose reabsorption via SGLT2 could elicit tubular damage in diabetic nephropathy through oxidative stress generation. So, SGLT2 inhibition by empagliflozin might protect proximal tubular cells from glycotoxicity in diabetic nephropathy partly via suppression of the AGE-RAGE-mediated oxidative stress generation. Given that tubulointerstitial injury is more important than glomerulopathy as a prognostic factor for diabetic nephropathy [4,5], inhibition 


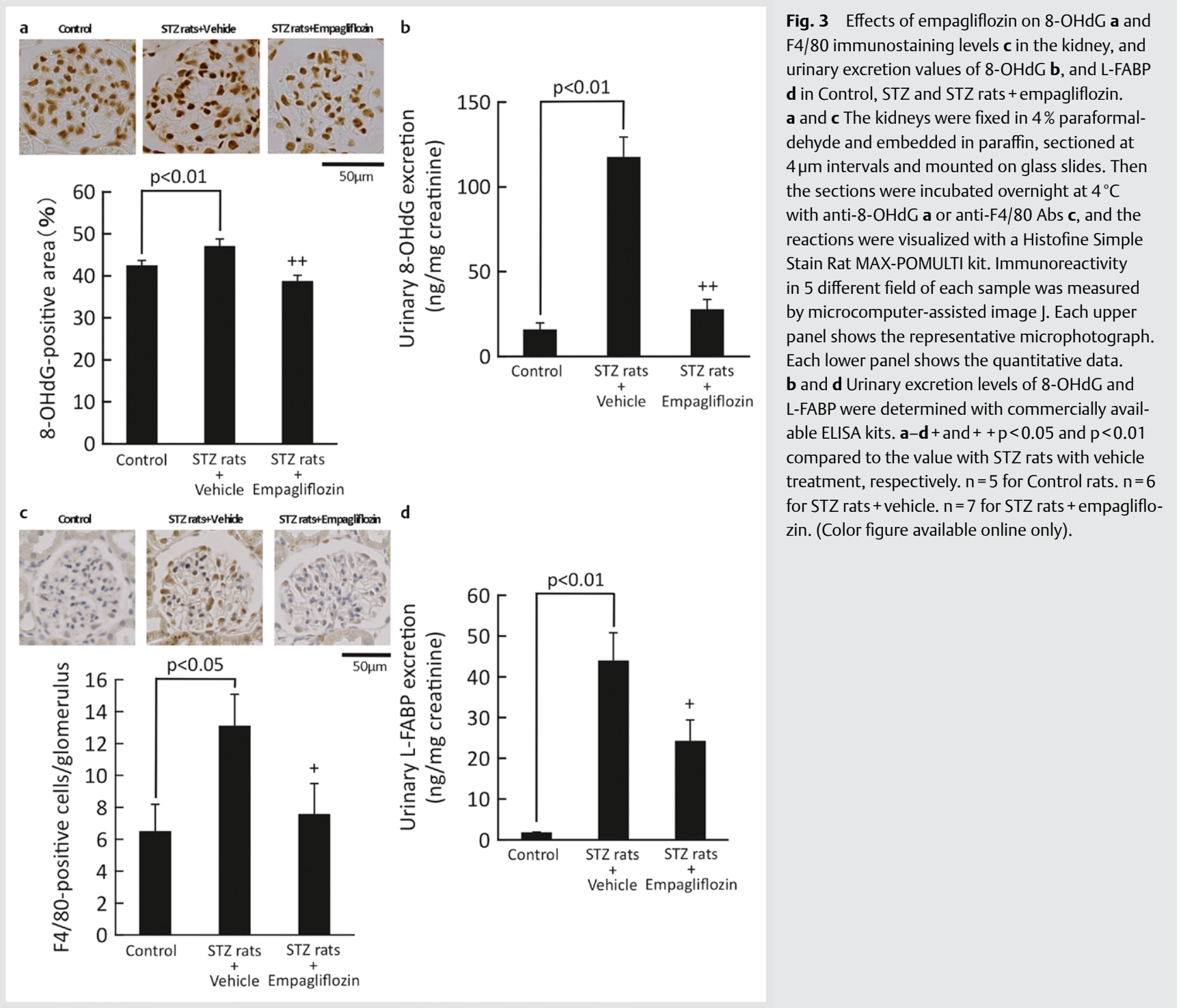

of SGLT2 might be a novel therapeutic target for preventing the progression of diabetic nephropathy [26]. Vallon et al. [27] recently showed that knockout of SGLT2 decreased hyperglycemia, but it did not attenuate kidney growth or the development of kidney inflammation, injury and fibrosis. Differences in method of SGLT2 suppression (empagliflozin vs. gene knockout) or animal species (SD rats vs. C57/BL/6 mice) could account for the discrepancies. Empagliflozin has been recently shown to reduce renal growth and inflammation in Akita mice of type 1 diabetes [28], thus supporting our speculation.

In the present study, empagliflozin treatment for 4 weeks lowered blood glucose levels, which might reduce the glomerular filtration of glucose in diabetic rats. This is one possible reason why glucosuria levels in empagliflozin-treated diabetic rats at 10 -week old were similar to those in vehicle-treated STZ rats at the same age despite the inhibition of glucose reabsorption by empagliflozin. Suppression of proximal tubular glucose reabsorption by empagliflozin might be balanced by its glomerular glucose filtration-reducing properties. Anyway, since there was no difference in glucosuria levels between empagliflozin- or vehicle-treated STZ rats, it is unlikely that luminal glucose delivery downstream of the proximal tubule could be involved in renal damage in our animal model.
In the present study, we found that inflammatory and fibrotic gene expression in the kidney of diabetic rats was significantly suppressed by empagliflozin. We, along with others, have previously shown that AGEs upregulate RAGE mRNA levels, and subsequently stimulates oxidative stress generation and increases gene expression of MCP-1, ICAM-1, PAI-1, TGF- $\beta$, and CTGF in human proximal tubular cells [29-31]. Therefore, the AGERAGE-induced oxidative stress generation could further potentiate the formation and accumulation of AGEs and subsequent RAGE overexpression. These positive feedback loops between AGEs and RAGE-downstream pathways might make a vicious cycle, thereby being involved in tubulointerstitial injury in diabetes. So, the present results indicate that the central mechanism for renoprotective properties of empagliflozin might be ascribed partly to its inhibitory actions on AGEs formation in proximal tubule, which is mediated by the suppression of glucose entry into tubular cells.

There is some controversy about the expression levels of SGLT2 in the diabetic kidney [32]. Indeed, SGLT2 expression was increased in the kidney of type 1 diabetic Akita mice or obese insulin resistant $\mathrm{db} / \mathrm{db}$ mice, whereas renal levels of SGLT2 were decreased or increased in STZ rats [22-34]. In this study, we found that although SGLT2 gene expression was unaltered under 

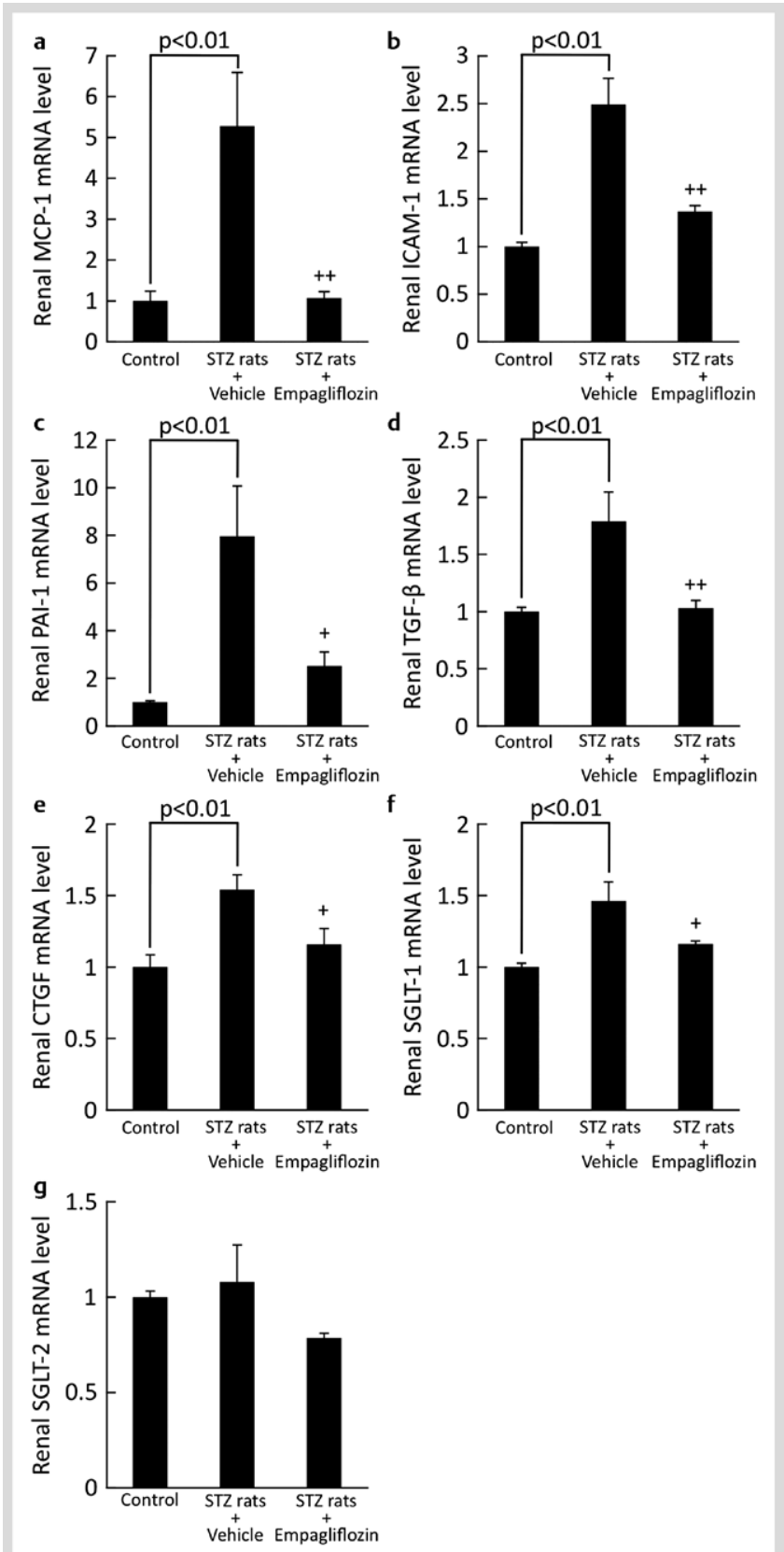

Fig. 4 Effects of empagliflozin on MCP- 1 a, ICAM- 1 b, PAI- 1 c, TGB- $\beta$ d, CTGF e, SGLT1 $\mathbf{f}$, and SGLT2 $\mathbf{g}$ gene expression in the kidney of Control, STZ, and STZ rats + empagliflozin. Total RNAs were extracted from the kidney of Control, STZ, and STZ rats + empagliflozin with RNAqueous4PCR kit. Quantitative real-time RT-PCR was performed. Data were normalized by the intensity of 185 mRNA-derived signals. $\mathbf{a}-\mathbf{g}+$ and ++ , $p<0.05$ and $p<0.01$ compared to the value with STZ rats with vehicle treatment, respectively. $n=5$ for Control rats. $n=6$ for STZ rats + vehicle. $\mathrm{n}=7$ for STZ rats + empagliflozin.

diabetic conditions irrespective of empagliflozin treatment, SGLT1 gene expression was increased in the kidney of STZ rats, which was completely prevented by empagliflozin. Under normal conditions, only small amount of the filtered glucose is taken up by SGLT1 [35]. However, SGLT1 has been recently shown to reabsorb about $70 \%$ of filtered glucose reaching the distal part of the proximal tubule under the conditions of complete SGLT2 inhibition [36]. Therefore, SGLT1 gene suppression by empagliflozin could potentiate its glucosuric and blood glucose-lowering properties in STZ rats.

\section{Limitations}

Since experimental animal model does not completely mimic human diabetic nephropathy, further prospective study is needed to clarify whether empagliflozin treatment could inhibit the AGE-RAGE axis and be superior to other oral hypoglycemic agents in protecting against tubular injury in human diabetic nephropathy.

\section{Acknowledgements}

$\nabla$

This study was supported in part by Grants-in-Aid for Scientific Research (B) from the Ministry of Education, Culture, Sports, Science and Technology, Japan (to S.Y.), and by MEXT-Supported Program for the Strategic Research Foundation at Private Universities, the Ministry of Education, Culture, Sports, Science and Technology (MEXT) (to S.Y.).

\section{Disclosure}

This project was financially supported by Boehringer Ingelheim. Dr. Yamagishi has received honoraria such as lecture fees from Boehringer Ingelheim and Eli Lilly.

\section{References}

1 Yamagishi S, Fukami K, Ueda S, Okuda S. Molecular mechanisms of diabetic nephropathy and its therapeutic intervention. Curr Drug Targets 2007; 8: 952-959

2 Karnib HH, Ziyadeh FN. The cardiorenal syndrome in diabetes mellitus. Diabetes Res Clin Pract 2010; 89: 201-208

3 Alsaad KO, Herzenberg AM. Distinguishing diabetic nephropathy from other causes of glomerulosclerosis: an update. J Clin Pathol 2007; 60: $18-26$

4 Taft JL, Nolan CJ, Yeung SP, Hewitson TD, Martin FI. Clinical and histological correlations of decline in renal function in diabetic patients with proteinuria. Diabetes 1994; 43: 1046-1051

5 Ziyadeh FN, Goldfarb S. The renal tubulointerstitium in diabetes mellitus. Kidney Int 1991; 39: 464-475

6 Vlassara $H$, Bucala $R$. Recent progress in advanced glycation and diabetic vascular disease: role of advanced glycation end product receptors. Diabetes 1996; (Suppl 3): S65-S66

7 Brownlee M, Cerami A, Vlassara H. Advanced glycosylation end products in tissue and the biochemical basis of diabetic complications. $\mathrm{N}$ Engl J Med 1988; 318: 1315-1321

8 Rahbar S. Novel inhibitors of glycation and AGE formation. Cell Biochem Biophys 2007; 48: 147-157

9 Yamamoto Y, Kato I, Doi T, Yonekura H, Ohashi S, Takeuchi M, Watanabe T, Yamagishi S, Sakurai S, Takasawa S, Okamoto H, Yamamoto $H$. Development and prevention of advanced diabetic nephropathy in RAGE-overexpressing mice. J Clin Invest 2001; 108: 261-268

10 Wendt TM, Tanji N, Guo J, Kislinger TR, Ou W, Lu Y, Bucciarelli LG, Rong LL, Moser B, Markowitz GS, Stein G, Bierhaus A, Liliensiek B, Arnold $B$, Nawroth PP, Stern DM, D'Agati VD, Schmidt AM. RAGE drives the development of glomerulosclerosis and implicates podocyte activation in the pathogenesis of diabetic nephropathy. Am J Pathol 2003; 162: $1123-1137$

11 Yamagishi S, Imaizumi T. Diabetic vascular complications: pathophysiology, biochemical basis and potential therapeutic strategy. Curr Pharm Des 2005; 11: 2279-2299

12 Yamagishi S, Nakamura K, Matsui T, Noda Y, Imaizumi T. Receptor for advanced glycation end products (RAGE): a novel therapeutic target for diabetic vascular complication. Curr Pharm Des 2008; 14: 487-495

13 Forbes JM, Cooper ME. Glycation in diabetic nephropathy. Amino Acids 2012; 42: 1185-1192 
14 Ojima A, Ishibashi Y, Matsui T, Maeda S, Nishino Y, Takeuchi M, Fukami $K$, Yamagishi S. Glucagon-like peptide-1 receptor agonist inhibits asymmetric dimethylarginine generation in the kidney of streptozotocin-induced diabetic rats by blocking advanced glycation end product-induced protein arginine methyltranferase- 1 expression. Am J Pathol 2013; 182: 132-141

15 Maeda S, Matsui T, Takeuchi M, Yoshida Y, Yamakawa R, Fukami K, Yamagishi S. Pigment epithelium-derived factor (PEDF) inhibits proximal tubular cell injury in early diabetic nephropathy by suppressing advanced glycation end products (AGEs)-receptor (RAGE) axis. Pharmacol Res 2011; 63: 241-248

16 Matsui T, Yamagishi S, Takeuchi M, Ueda S, Fukami K, Okuda S. Nifedipine inhibits advanced glycation end products (AGEs) and their receptor (RAGE) interaction-mediated proximal tubular cell injury via peroxisome proliferator-activated receptor-gamma activation. Biochem Biophys Res Commun 2010; 398: 326-330

17 Lee YJ, Lee YJ, Han HJ. Regulatory mechanims of $\mathrm{Na}^{+} /$glucose cotransporters in renal proximal tubule cells. Kidney Int 2007; 72: 527-535

18 Sabolic I, Vrhovac I, Eror DB, Gerasimova M, Rose M, Breljak D, Ljubojevic M, Brzica H, Sebastiani A, Thal SC, Sauvant C, Kipp H, Vallon V, Koepsell $H$. Expression of $\mathrm{Na}+$-D-glucose cotransporter SGLT2 in rodents is kidney-specific and exhibits sex and species differences. Am J Physiol Cell Physiol 2012; 302: C1174-C1188

19 Santer $R$, Calado J. Familial renal glucosuria and SGLT2: from a mendelian trait to a therapeutic target. Clin J Am Soc Nephrol 2010; 5: 133-141

20 Bailey CJ, Gross JL, Pieters A, Bastien A, List JF. Effect of dapagliflozin in patients with type 2 diabetes who have inadequate glycemic control with metformin: a randomized, double-blind, placebo-controlled trial. Lancet 2010; 375: 2223-2233

21 Maeda S, Matsui T, Takeuchi M, Yamagishi S. Sodium-glucose cotransporter 2-mediated oxidative stress augments advanced glycation end products-induced tubular cell apoptosis. Diabetes Metab Res Rev 2013; 29: 406-412

22 Takeuchi M, Makita Z, Bucala R, Suzuki T, Koike T, Kameda Y. Immunological evidence that non-carboxymethyllysine advanced glycation end-products are produced from short chain sugars and dicarbonyl compounds in vivo. Mol Med 2000; 6: 114-125

23 Panchapakesan $U$, Pegg $K$, Gross S, Komala MG, Mudaliar H, Forbes J, Pollock C, Mather A. Effects of SGLT2 inhibition in human kidney proximal tubular cells - renoprotection in diabetic nephropathy? PLoS One 2013; 8: e54442

24 Osorio H, Coronel I, Arellano A, Pacheco U, Bautista R, Franco M, Escalante $B$. Sodium-glucose cotransporter inhibition prevents oxidative stress in the kidney of diabetic rats. Oxid Med Cell Longev 2012; 542042
25 Morrisey $K$, Steadman $R$, Williams JD, Phillips AO. Renal proximal tubular cell fibronectin accumulation in response to glucose is polyol pathway dependent. Kidney Int 1999; 55: 160-167

26 Gilbert RE. Sodium-glucose linked transporter-2 inhibitors: potential for renoprotection beyond blood glucose lowering? Kidney Int 2014; 86: $693-700$

27 Vallon V, Rose M, Gerasimova M, Satriano J, Platt KA, Koepsell H, Cunard $R$, Sharma K, Thomson SC, Rieg T. Knockout of Na-glucose transporter SGLT2 attenuates hyperglycemia and glomerular hyperfiltration but not kidney growth or injury in diabetes mellitus. Am J Physiol Renal Physiol 2013; 304: F156-F167

28 Vallon V, Gerasimova M, Rose MA, Masuda T, Satriano J, Mayoux E Koepsell H, Thomson SC, Rieg T. SGLT2 inhibitor empagliflozin reduces renal growth and albuminuria in proportion to hyperglycemia and prevents glomerular hyperfiltration in diabetic Akita mice. Am J Physiol Renal Physiol 2014; 306: F194-F204

29 Matsui T, Yamagishi S, Takeuchi M, Ueda S, Fukami K, Okuda S. Irbesartan inhibits advanced glycation end product (AGE)-induced proximal tubular cell injury in vitro by suppressing receptor for AGEs (RAGE) expression. Pharmacol Res 2010; 61: 34-39

30 Ishibashi Y, Matsui T, Takeuchi M, Yamagishi S. Metformin inhibits advanced glycation end products (AGEs)-induced renal tubular cell injury by suppressing reactive oxygen species generation via reducing receptor for AGEs (RAGE) expression. Horm Metab Res 2012; 44: 891-895

31 Chung AC, Zhang H, Kong YZ, Tan JJ, Huang XR, Kopp JB, Lan HY. Advanced glycation end-products induce tubular CTGF via TGF-betaindependent Smad3 signaling. J Am Soc Nephrol 2010; 21: 249-260

32 Vallon $V$. The proximal tubule in the pathophysiology of the diabetic kidney. Am J Physiol Renal Physiol 2011; 300: R1009-R1022

33 Albertoni Borghese MF, Majowicz MP, Ortiz MC, Passalacqua Mdel R, Sterin Speziale NB, Vidal NA. Expression and activity of SGLT2 in diabetes induced by streptozotocin: relationship with the lipid environment. Nephron Physiol 2009; 112: 45-52

34 Osorio H, Coronel I, Arellano A, Franco M, Escalante B, Bautista R. Ursodeoxycholic acid decreases sodium-glucose cotransporter (SGLT2) expression and oxidative stress in the kidney of diabetic rats. Diabetes Res Clin Pract 2012; 97: 276-282

35 Abdul-Ghani YMA, DeFronzo RA, Norton L. Novel hypothesis to explain why SGLT2 inhibitors inhibit only $30-50 \%$ of filtered glucose load in humans. Diabetes 2013; 62: 3324-3328

36 Powell DR, DaCosta CM, Gay J, Ding ZM, Smith M, Greer J, Doree D, JeterJones $S$, Mseeh F, Rodriguez LA, Harris A, Buhring L, Platt KA, Vogel $P$, Brommage R, Shadoan MK, Sands AT, Zambrowicz B. Improved glycemic control in mice lacking Sglt1 and Sglt2. Am J Physiol Endocrinol Metab 2013; 304: E117-E130 